Bull. Austral. Math. Soc.

$57 \mathrm{Q} 91,57 \mathrm{~s} 99$

VOL. 65 (2002) [45-53]

\title{
CW DECOMPOSITIONS OF EQUIVARIANT CW COMPLEXES
}

\author{
M. Cencelj and N. Mramor Kosta
}

\begin{abstract}
We discuss conditions which ensure that a $G$-CW complex is $G$-homotopy equivalent to a $\mathrm{CW}$ complex with cellular action with respect to some $\mathrm{CW}$ decomposition of the compact Lie group $G$. For $G=S U(2)$, we prove that for every $G$-CW complex $X$, there exists a CW complex $Y$ which is $G$-homotopy equivalent to $X$, such that the action $G \times Y \rightarrow Y$ is a cellular map.
\end{abstract}

\section{INTRODUCTION}

Let $G$ be a compact Lie group. A $G$-cell of dimension $n$ is a space of the form $G / H \times D^{n}$, where $H$ is a closed subgroup of $G$ and $D^{n}$ is an $n$-cell. A $G-C W$ complex $X$ (or an equivariant $C W$ complex in the terminology of [9]) is constructed by iterated attaching of $G$-cells. It is the union of $G$-spaces $X^{(n)}$ such that $X^{(0)}$ is a disjoint union of $G$-cells of dimension 0 , that is, orbits $G / H$, and $X^{(n+1)}$ is obtained from $X^{(n)}$ by attaching $G$-cells of dimension $n+1$ along equivariant attaching maps $G / H \times \partial D^{n+1} \rightarrow X^{(n)}$. The space $X^{(n)}$, which is called the $n$-skeleton of $X$, is thus the union of all $G$-cells of dimension at most $n$ (the topological dimension of $X^{(n)}$ is in general greater than $n$ ). For basic facts about $G$-complexes see the original papers [5] and [3] or the exposition in [9].

For discrete groups $G$ it is well known that every $G-\mathrm{CW}$ complex is also a $\mathrm{CW}$ complex with a cellular action of $G$ (this follows for example from [9, Proposition 1.16, p. 102]). For non-discrete groups, Illman [4] gave an example showing that a $G-C W$ complex $X$ does not always admit a CW decomposition, compatible with the given $G$ $\mathrm{CW}$ decomposition, and proved that there always exists a homotopy equivalent $\mathrm{CW}$ complex $Y$ which is finite if $X$ is a finite $G$-complex.

In this paper we consider the following problem. Given a $G$-CW complex $X$, does there exist a $G$-space $Y, G$-homotopy equivalent to $X$, with a $\mathrm{CW}$ decomposition such that the action $\rho: G \times Y \rightarrow Y$ is a cellular map with respect to some decomposition of $G$. The existence of such a $Y$ is interesting from the point of view of equivariant homology and cohomology. For example, Greenlees and May showed that for some groups $G$ the generalised Tate cohomology defined in [1] can be calculated from the CW decomposition

Received 19th April, 2001

Partially supported by Ministry of Science and Technology of the Republic of Slovenia Research Grant No. J1-0885-0101-98.

Copyright Clearance Centre, Inc. Serial-fee code: 0004-9727/02 $\$ A 2.00+0.00$. 
of $Y$. Also, the Borel equivariant cohomology $H_{G}^{*}(X)=H^{*}\left(E G \times_{G} X\right)$ of a $G$-CW complex $X$ can be computed using the cellular cohomology of the CW complex $Y$ which is $G$-homotopy equivalent to $E G \times_{G} X$.

In general, it is not known to the authors if, for a given group $G$, every $G$-CW complex is $G$-homotopy equivalent to a $\mathrm{CW}$ complex $Y$ with the required properties. For $G=S^{1}$, Greenlees and May [1, Lemma 14.1] gave a construction of $Y$ for any $X$. In case of non-Abelian groups, the construction of $Y$ is more difficult, since the fixed point sets $(G / H)^{K}$ of actions of subgroups $K<G$ on the orbits $G / H$ are in general nontrivial. In [7], the two non-Abelian 1-dimensional compact Lie groups, the orthogonal group $O(2)$ and the continuous quaternionic group $N_{S U(2)} T$, are considered but the construction of $Y$ rests on a property of these two groups which is satisfied only for a few particular groups $G$. In this paper we consider the 3-dimensional group $G=S U(2)$.

The paper is organised as follows. In Section 2, two conditions on the set of isotropy subgroups of a $G-C W$ complex $X$ which enable the construction of a $G$-homotopy equivalent CW complex $Y$ by induction on the $G$-skeletons of $X$ are stated. We show that the class of $G$-CW complexes with finitely many isotropy types satisfies these two conditions. We also show that if a group $G$ has the property that the set of all closed subgroups satisfies these two conditions, then every $G$-CW complex has a $G$-homotopy equivalent CW complex with a cellular action of $G$. In Section 3, the actions of subgroups of the group $S U(2)$ on orbits of $S U(2)$ are analysed. We show that a set of closed subgroups of $S U(2)$ satisfies the two conditions of Section 2 and as a result obtain our main theorem.

THEOREM 1. Any $S U(2)-C W$ complex $X$ is G-homotopy equivalent to a $C W$ complex $Y$ which is an $S U(2)$-space with a cellular action of $S U(2)$.

Finally, in Section 4 some other examples are discussed.

\section{REPRESENTATIVE FAMILIES OF SUBGROUPS}

Let $G$ be a compact Lie group, $X$ a $G$-CW complex, and $\mathcal{H}$ the family of isotropy subgroups of the action.

For a $G$-CW complex $X$, a representative family $\mathcal{K}$ of isotropy subgroups is a family of closed subgroups of $G$ such that each isotropy subgroup of $X$ is conjugate to a member of $\mathcal{K}$. We shall call a representative family good with respect to a given $\mathrm{CW}$ decomposition of $G$ if the following two conditions are satisfied.

(1) For each $H \in \mathcal{K}$, there is a CW decomposition of $G / H$ with respect to which the action $\mu: G \times G / H \rightarrow G / H$ is cellular.

(2) For each $K \in \mathcal{K}$, the fixed point set $(G / H)^{K}$ is a subcomplex of the CW complex $G / H$.

Let us first prove that the existence of a good representative family suffices for the construction of a $G$-homotopy equivalent $\mathrm{CW}$ complex $Y$. 
Proposition 1. Let $G$ be a compact Lie group with a given $C W$ decomposition, $X$ a $G-C W$ complex and $\mathcal{K}$ a good representative family of isotropy subgroups. Then there exists a $C W$ complex $Y$ with a cellular action of $G$ and a $G$-homotopy equivalence $h: X \rightarrow Y$.

Proof: Following [1, Lemma 14.1], we shall construct a CW complex $Y$ and a $G$-homotopy equivalence $h: X \rightarrow Y$ by induction on the $G$-skeletons $X^{(i)}$ of $X$.

Since every isotropy subgroup of $X$ is conjugate to a member of $\mathcal{K}$, the 0 -skeleton $X^{(0)}$ is homeomorphic to a disjoint union of orbits $G / H_{i}$, where $H_{i} \in \mathcal{K}$. Since $\mathcal{K}$ is good, every orbit $G / H$ has a $\mathrm{CW}$ decomposition satisfying conditions (1) and (2). Let $Y_{0}$ be $X^{(0)}$ with this CW decomposition on every $G$-cell $G / H_{i}$. Because of condition (1), the action $\mu: G \times Y_{0} \rightarrow Y_{0}$ is cellular, and because of condition (2),

$$
\left(Y_{0}\right)^{K}=\coprod\left(G / H_{i}\right)^{K}
$$

is a subcomplex of $Y_{0}$ for every $K \in \mathcal{K}$. The $G$-homotopy equivalence on the 0 -skeleton is the identity $h_{0}=\mathrm{id}: X^{(0)} \rightarrow Y_{0}$.

By induction we assume that there exist a $\mathrm{CW}$ complex $Y_{n-1}$ with a cellular action of $G$, such that for every $K \in \mathcal{K}$ the fixed point set $\left(Y_{n-1}\right)^{K}$ is a subcomplex, and a $G$-homotopy equivalence

$$
h_{n-1}: X^{(n-1)} \rightarrow Y_{n-1} .
$$

For any $G$-cell $e_{\nu}^{n} \in X^{(n)}$, the attaching $G$-map $G / H_{\nu} \times S^{n-1} \rightarrow X^{(n-1)}$ is determined by its restriction

$$
\varphi_{\nu}: S^{n-1} \rightarrow\left(X^{(n-1)}\right)^{H_{\nu}} .
$$

Let $\psi_{\nu}$ be a non-equivariant cellular approximation of the composition

$$
h_{n-1} \circ \varphi_{\nu}: S^{n-1} \rightarrow\left(Y_{n-1}\right)^{H_{\nu}} .
$$

Since the action of $G$ on $Y_{n-1}$ is cellular, the natural $G$-extension

$$
\tilde{\psi}_{\nu}: G / H_{\nu} \times S^{n-1} \rightarrow Y_{n-1}
$$

of $\psi_{\nu}$ is also cellular, and the space

$$
Y_{n}=\coprod_{e_{\nu}^{n} \in X^{(n)}}\left(G / H_{\nu} \times D^{n}\right) \cup_{\amalg \tilde{\psi}_{\nu}} Y_{n-1}
$$

is a CW complex with a cellular action of $G$. For each $K \in \mathcal{K}$, the fixed point set $\left(Y_{n}\right)^{K}$ is obtained by gluing the subcomplexes $\left(G / H_{\nu}\right)^{K} \times D^{n}$, corresponding to the $n$-cells, and the subcomplex $\left(Y_{n-1}\right)^{K}$ along a cellular map. So $\left(Y_{n}\right)^{K}$ is a subcomplex of $Y_{n}$. The $G$-homotopy $h_{n}$ is obtained so that $h_{n-1}$ is extended $G$-cell by $G$-cell over the whole space $Y_{n}$. In the direct limit, we obtain the desired CW complex $Y$ and $G$-homotopy equivalence $h$. 
For example, for any compact Lie group $G$, every $G$-CW complex which consists of a free part and a part which is fixed by the action is $G$-homotopy equivalent to a $\mathrm{CW}$ complex with a cellular action of $G$. More generally,

Proposition 2. For every G-CW complex with a finite representative family of isotropy groups $\mathcal{K}$ which satisfies condition (1) there exists a $G$-homotopy equivalent $C W$ complex $Y$ with a cellular action of $G$.

Proof: By assumption, condition (1) is satisfied. The following lemma shows that condition (2) is also satisfied, so the family $\mathcal{K}$ is good.

LEMma 1. If for a given $H \in \mathcal{K}$ the collection of fixed point sets $(G / H)^{K}, K \in \mathcal{K}$, is a finite family of subsets of $G / H$, then the orbit $G / H$ has a $C W$-decomposition with respect to which every fixed point set $(G / H)^{K}, K \in \mathcal{K}$, is a subcomplex.

Proof: For every $K \in \mathcal{K}$ the orbit $(G / H)$ is a smooth $K$-manifold, and the fixed point set $(G / H)^{K}$ is a submanifold [9, p. 42] which is nontrivial only if $K$ is conjugate to a subgroup of $H$. The family $\left\{(G / H)^{K}, K \in \mathcal{K}\right\}$ is a finite family of smooth submanifolds of $G / H$ which, by the differentiable slice theorem (compare for example [2, Theorem I.5]), intersect transversally. By $[6,10.11,10.14]$, this implies that there exists a CW decomposition of $G / H$ such that each $(G / H)^{K}, K \in \mathcal{K}$, is a subcomplex.

COROLLARY If there exists a good representative family of all closed subgroups of a compact Lie group $G$, then every $G$-CW complex $X$ has a $G$-homotopy equivalent $C W$ complex $Y$ with a cellular action of $G$.

Proof: This follows immediately from Proposition 1.

\section{A GOOD REPRESENTATIVE FAMILY FOR $S U(2)$}

Let $G$ be $S U(2) \cong S p(1)$. An element $x \in G$ can be represented in the form

$$
x=\left[\begin{array}{cc}
z_{1} & z_{2} \\
-\bar{z}_{2} & \bar{z}_{1}
\end{array}\right], \quad\left|z_{1}\right|^{2}+\left|z_{2}\right|^{2}=1, \quad z_{1}, z_{2} \in \mathbb{C}
$$

or as the unit quaternion $q=z_{1}+j z_{2}$. The centre $Z_{G}$ is generated by $-I \in S U(2)$ (or $-1 \in S p(1)$ ), the only element of order 2 . The projection $G \rightarrow G / Z_{G} \cong S O(3)$ associates to a unit quaternion written in polar form as $q=(\cos \varphi, j \mathbf{e})$ the rotation with axis $\mathbf{e} \in \mathbb{R}^{3}$ through the angle $\varphi$.

The isomorphism classes of closed subgroups of $S U(2)$ are known. Since there are no non-Abelian 2-dimensional Lie groups, the dimension of a proper closed subgroup is at most 1. The 0 - and 1-dimensional subgroups are ([10, p. 155]: [8, p. 404]):

1. the circle group $\mathbb{T}$, which is a maximal torus;

2. the normaliser $N T=N_{S U(2)} T$ of a maximal torus; 
3. a cyclic group $\mathbb{Z} / n$;

4. the quaternionic group

$$
\left\langle x, y \mid x^{2}=y^{2}, y^{-1} x y=x^{-1}\right\rangle
$$

or a generalised quaternionic group

$$
\left\langle x, y \mid x^{n}=y^{2}, y^{-1} x y=x^{-1}\right\rangle \text {; }
$$

5. the special linear group $S L_{2}\left(\mathbb{F}_{3}\right)$, which is a lift of the tetrahedral subgroup of $S O(3)$;

6. the special linear group $S L_{2}\left(\mathbb{F}_{5}\right)$, which is a lift of the icosahedral subgroup of $S O(3)$;

7. a lift of the octahedral subgroup of $S O(3)$, which is an extension of the symmetric group $S_{4}$.

Let the representative family $\mathcal{K}$ consist of the following closed subgroups of $S U(2)$.

1. The conjugacy class of maximal tori is represented by the group of real rotations

$$
S O(2)=\left\{a_{t}=\left[\begin{array}{rr}
\cos t & -\sin t \\
\sin t & \cos t
\end{array}\right], \quad t \in \mathbb{R}\right\} .
$$

2. The conjugacy class of normalisers of maximal tori is represented by $N S O(2)$ which is generated by $S O(2)$ and the element

$$
u=\left[\begin{array}{ll}
0 & i \\
i & 0
\end{array}\right]
$$

3. The cyclic groups $\mathbb{Z} / n$ are represented by subgroups $C_{n}<S O(2)$, generated by rotations $a_{2 \pi / n}$. In a group of rank 1 , a cyclic group of order $n$ is completely determined by the maximal torus in which it lies, and so, since the maximal tori are all conjugate, every cyclic subgroup of order $n$ is conjugate to $C_{n}$.

4. The generalised quaternionic groups are represented by subgroups $G_{2 n}<$ $N S O(2)$, where the generator $x$ is the rotation $a_{\pi / n}$ and $y$ is $u$. Let us show that there is only one conjugacy class of groups isomorphic to $G_{2 n}$ in $S U(2)$. Every subgroup $H=\langle x, y\rangle \cong G_{2 n}$ of $S U(2)$ is contained in the normaliser $N T$ of some maximal torus $T$, more precisely in $N T=T \cdot y$, where $T$ is the maximal torus through $x$. Since all normalisers of maximal tori are conjugate, we can assume that $H<N S O(2)$. In this case, $x \in S O(2)$, and $y$ is in the non-identity component of $N S O(2)$. All elements of the non-identity component of $N S O(2)$ are of the form

$$
u(t)=\left[\begin{array}{cc}
i \cos t & i \sin t \\
i \sin t & -i \cos t
\end{array}\right]
$$


and are of order 4 . For every $t$, the group $H(t)=\left\langle a_{2 \pi / n}, u(t)\right\rangle \cong G_{2 n}$, and is conjugate to $G_{2 n}$ by the element $u(t / 2)$.

5. The remaining three groups have only one conjugacy class each in $S U(2)$, since their projections to $S O(3)$, the symmetry groups of the tetrahedron, octahedron or icosahedron, have only one conjugacy class each in $S O(3)$. Two copies, $H_{1}$ and $H_{2}$, of the same symmetry group in $S O(3)$ are conjugate by the matrix describing the change of basis which takes the polyhedron fixed by $H_{1}$ to the polyhedron fixed by $H_{2}$. An obvious choice for the representative of $S L_{2}\left(\mathbb{F}_{3}\right)$ is $N G_{4}$. For the remaining two groups, any choice of representatives is good.

Proposition 3. The family $\mathcal{K}$ is a good representative family of conjugacy classes of all closed subgroups of $S U(2)$ with respect to the standard decomposition of $S U(2)$ into one 0 -cell and one 3-cell.

Proof: Let us first prove that the representative family $\mathcal{K}$ satisfies condition (1).

LEMma 2. If $G=S U(2)$ is given the standard $C W$ decomposition into one 0-cell and one 3-cell and $H<G$ is a closed subgroup, then for any $C W$ decomposition of the orbit $G / H$, the action $\mu: G \times G / H \rightarrow G / H$ is cellular.

Proof: Choose $e^{0}=I \in S U(2)$. For any closed subgroup $H$, the quotient $G / H$ is a connected manifold of dimension 2 or 3 . For any CW decomposition of $G / H$, the 0,1 and 2 skeletons of $G \times G / H$ consist of cells of the form $e^{0} \times f_{\nu}^{j}$, where $f_{\nu}^{j}$ is a $j$-cell of $G / H$, and $j=0,1$ or 2 . Since multiplication by $e^{0}=I$ is the identity,

$$
\mu\left(e^{0} \times f_{\nu}^{j}\right)=f_{\nu}^{j} \subset(G \times G / H)^{(j)} .
$$

For $j \geqslant 3$, the $j$-skeleton of $G \times G / H$ is mapped to $G / H=(G / H)^{(3)}$.

This implies that it suffices to find a $C W$ decomposition for every orbit $G / H, H \in \mathcal{K}$, such that all fixed point sets $(G / H)^{K}, K \in \mathcal{K}$, are subcomplexes. In order to prove this we shall show that for every $H \in \mathcal{K}$ the family $\left\{(G / H)^{K}, K \in \mathcal{K}\right\}$ of fixed point sets is a finite family of subsets of $G / H$. By Lemma 1 it follows that the family $\mathcal{K}$ is good.

The fixed point set of the action of $K$ on $G / H$ can be described as

$$
(G / H)^{K}=\left\{g H \mid g^{-1} K g<H\right\} .
$$

It is nontrivial only if $K$ is subconjugate to $H$. So, for every $H \in \mathcal{K}$, it suffices to consider the subgroups $K \in \mathcal{K}$ which are subconjugate to $H$.

If $H$ is a finite group, it has only finitely many subconjugate groups, so the family of fixed point sets $(G / H)^{K}$ is finite. It remains to consider the two 1-dimensional groups in $\mathcal{K}$.

The only nontrivial groups $K \in \mathcal{K}$ subconjugate to $S O(2)$ are $S O(2)$ and the cyclic groups $C_{n}$. A short computation shows that for every $n^{*} \neq 2$,

$$
(G / S O(2))^{C_{n}}=N S O(2) / S O(2)=\mathbb{Z} / 2 .
$$


If $n=2$, then $C_{2}$ is the centre $Z_{G}$, and $(G / S O(2))^{C_{2}}=G / S O(2)$. The family $\left\{(G / S O(2))^{K}, K \in \mathcal{K}\right\}$ therefore has two members: the whole space $G / S O(2)$ and $N S O(2) / S O(2)$.

A nontrivial group $K \in \mathcal{K}$ subconjugate to $N S O(2)$ is either a cyclic group $C_{n}$, a quaternionic group $G_{2 n}, S O(2)$, or the whole group $N S O(2)$. For $K=C_{n}, n \neq 2,4$, every subgroup of $N S O(2)$ conjugate to $C_{n}$ must be contained in $S O(2)$, since every element $u(t)$ of the non-unit component of $N S O(2)$ has order 4. So $K=C_{n}$. Any conjugation $c_{g}: G \rightarrow G$ which maps $C_{n}$ into $N S O(2)$ must therefore map the generator of $C_{n}$ to an element of $C_{n}$. So

$$
(G / N S O(2))^{C_{n}}=(G / N S O(2))^{S O(2)}=N S O(2) / N S O(2)
$$

is a point. The group $C_{4}$ is conjugate to every cyclic subgroup of $N S O(2)$ generated by an element $u(t)$, and

$$
(G / N S O(2))^{C_{4}}=\left\{g N S O(2) \mid g^{-1} a_{\pi / 2} g=u(t) \text { for some } t\right\} .
$$

For $n=2,(G / N S O(2))^{C_{2}}=G / N S O(2)$. The only subgroup of $N S O(2)$ conjugate to $G_{2 n}$ is $G_{2 n}$, and any conjugation $c_{g}: G \rightarrow G$ which maps $G_{2 n}$ into $N S O(2)$ must preserve the subgroup $C_{n}$, and it must map $u$ into some element $u(t)$. A simple computation shows that this is true for every $g \in N S O(2)$. On the other hand, it is not true if $g \notin N S O(2)$, since no such element preserves rotations. So, $(G / N S O(2))^{G_{2 n}}=N S O(2) / N S O(2)$ is a point for all $n$. The remaining finite three subgroups in $\mathcal{K}$ are not isomorphic to any subgroup of $N S O(2)$. The family $(G / N S O(2))^{K}, K \in \mathcal{K}$, therefore has three members.

Proof of Theorem 1: Since we have found a representative family for the family of all closed subgroups of $S U(2)$ which is good with respect to the standard CW decomposition of the group $S U(2)$, the theorem follows from the Corollary.

\section{SOME EXAMPLES}

In this section, we give several examples concerning a question posed in [7]. In [7] it is proved that for $G=O(2)$ or $G=N_{S U(2)} T$, a $G$-CW complex is $G$-homotopy equivalent to a CW complex with cellular action of $G$. The proof rests on the following property of these two 1-dimensional groups. The natural projection $\pi$ from the set of all closed subgroups $\mathcal{S}(G)$ to the set of all conjugacy classes of closed subgroups $\mathcal{C}(G)$ has a section $\nu: \mathcal{C}(G) \rightarrow \mathcal{S}(G)$ such that if $(H)<(K)$ then $\nu((H))<\nu((K))$, where the notation $(H)<(K)$ means that $H$ is subcojugate to $K$. The question of necessary and sufficient conditions for the existence of such a $\nu$ is posed.

Let us first show that this condition is not satisfied in $S U(2)$. Let us pick any representative $T$ for the maximal torus. A representative for the conjugacy class of $G_{4}$ must contain the representative $H_{4}<T$ for $\mathbb{Z} / 4$. Once a representative for $G_{4}$ is chosen, 
it determines the representative for $N G_{4} \cong S L_{2}\left(\mathbb{F}_{3}\right)$, which also contains $H_{4}<T$ but no cyclic groups $\mathbb{Z} / n \cong H_{n}<T, n>4$. Specifically, it does not contain the representative for the conjugacy class $\mathbb{Z} / 6$. On the other hand, $S L_{2}\left(\mathbb{F}_{3}\right)$ has elements of order 6 , so it contains a copy of $\mathbb{Z} / 6$. Therefore, $\mathbb{Z} / 6$ is subconjugate to $N G_{4}$, but the representatives $\mathrm{H}_{6}$ and $\mathrm{NH}_{4}$ for the conjugacy classes of these two groups in $S U(2)$ cannot be chosen so that $\mathrm{H}_{6}<\mathrm{NH}_{4}$.

A similar argument shows that, although the tetrahedral group contains a copy of $\mathbb{Z} / 3$, we cannot choose a representative for the conjugacy class of $\mathbb{Z} / 3$ which would be contained in the conjugacy class of the tetrahedral group in $S O(3)$.

It follows that no compact Lie group containing either $S U(2)$ or $S O(3)$ has a section with the required properties.

Here is an example of a finite group which does not have a section with the required properties. The authors would like to thank Aleš Vavpetič for pointing out this example. Let $S_{7}$ be the symmetric group on 7 letters. We have the following subgroups.

1. $H_{2}=Z / 2$, generated by one transposition,

2. $H_{3}=Z / 3$ generated by a 3 -cycle,

3. $H_{4}=Z / 4$ generated by a 4 -cycle,

4. $H_{6}=Z / 2 \times Z / 3$ generated by a transposition and a disjoint 3-cycle,

5. $H_{8}=Z / 2 \times Z / 4$ generated by a transposition and a disjoint 4-cycle,

6. $H_{12}=Z / 3 \times Z / 4$ generated by a 3 -cycle and a disjoint 4-cycle.

Assume that $\langle(123),(4567)\rangle$ is the representative of $H_{12}$. Since $H_{3}<H_{12}$, we must pick $\langle(123)\rangle$ for $H_{3}$, and for the same reason, $\langle(4567)\rangle$ for $H_{4}$. Since $H_{3}<H_{6}$, the representative for $H_{6}$ must contain the 3-cycle (123), and the transposition generating $H_{2}$ must be some $(a b)$, where $a, b \in\{4, \ldots, 7\}$. Since $H_{2}<H_{6}$, where $\langle(a b)\rangle$ is the representative for $H_{2}$, then for $H_{8}$ the only possibility is $\langle(a b),(4567)\rangle$. But $(a b)$ is not disjoint to $(4567)$, and so $\langle(a b),(4567)\rangle$ is not isomorphic to $H_{8}$.

Furthermore, it obviously follows that no finite group containing $S_{7}$ has the required section.

\section{REFERENCES}

[1] J.P.C. Greenlees and J.P. May, Generalized Tate cohomology, Memoirs Amer. Math. Soc. 113 (American Mathematical Society, Providence, R.I., 1995).

[2] W.Y. Hsiang, Cohomology theory of topological transformation groups (Springer-Verlag, Berlin Heidelberg New York, 1975).

[3] S. Illman, 'Equivariant singular homology and cohomology for actions of compact Lie groups', in Proceedings of the Second Conference on Compact Transformation Groups, Univ. of Massachusetts, Amherst, 1971, Lecture Notes in Math. Vol. 298 (Springer-Verlag, Berlin, Heidelberg, New York, 1972), pp. 403-415.

[4] S. Illman, 'Restricting the transformation group in equivariant CW complexes', Osaka J. Math. 27 (1990), 191-206. 
[5] T. Matumoto, 'On G-CW complexes and a theorem of J.H.C. Whitehead', J. Fac. Sci. Univ. Tokyo Sect. 1A Math. 18 (1971), 109-125.

[6] J.R. Munkres, Elementary differential topology, Annals of Mathematical Studies 54 (Princeton University Press, Princeton, New Jersey, 1966).

[7] J.A. Pérez, 'Substitutional lemma for $G$-spaces of 1-dimensional groups', Glasgow Math. J. 38 (1996), 215-220.

[8] I. Suzuki, Group Theory I (Springer-Verlag, Berlin, Heidelberg, New York, 1982).

[9] T. tom Dieck. Transformation groups (Walter de Gruyter, Berlin, NewYork, 1987).

[10] T. tom Dieck, Transformation groups and representation theory, Lecture Notes in Mathematics 766 (Springer-Verlag, Berlin, Heidelberg, New York, 1979).

Institute of Mathematics, Physics and Mechanics,

Faculty of Computer and Information Science and Faculty of Education

University of Ljubljana

Jadranska 19

SI-1000 Ljubljana

Slovenia

e-mail: neza.mramor-kosta@fmf.uni-1j.si

matija.cencelj@fmf.uni-lj.si 